\title{
Division of Blood Diseases and Resources
}

National Cancer Institute

\section{Source}

National Cancer Institute. Division of Blood Diseases and Resources. NCI Thesaurus.

Code C82614.

A division of the National Heart, Lung, and Blood Institute that supports research on the causes, prevention, and treatment of nonmalignant blood diseases, including anemias, sickle cell disease, and thalassemia; premalignant processes such as myelodysplasia and myeloproliferative disorders; hemophilia and other abnormalities of hemostasis and thrombosis; and immune dysfunction. 